\title{
In-hospital stroke
}

\author{
Kolja Schürmann ${ }^{7}$ and Arno Reich ${ }^{1^{*}}$
}

1Department of Neurology, University Hospital Rheinisch-Westfälische Technische Hochschule [RWTH] Aachen, Aachen, Germany

Article Info

\section{Article Notes}

Received: January 19, 2017

Accepted: February 17, 2017

\section{*Correspondence:}

Arno Reich (MD), Department of Neurology, RWTH Aachen University, Pauwelsstrasse 30, D-52074 Aachen, Germany

Email: areich@ukaachen.de

C) 2016 Reich A. This article is distributed under the terms of the Creative Commons Attribution 4.0 International License

\section{Keywords}

In-hospital stroke (IHS)

Community onset stroke (COS)

Intravenous recombinant tissue plasminogen activator (IVRTPA) Neurothrombectomy

\begin{abstract}
In-hospital stroke (IHS), which represents between $2.2 \%$ and $17 \%$ of all strokes, differs from community-onset stroke (COS) in etiology, treatment approaches and outcome. Patients hospitalized for cardiac disease are especially vulnerable to predominantly cardioembolic IHS. Stroke severity, functional outcome and mortality compare unfavorably to COS. Difficulties in symptom recognition, intra-hospital delays, various stroke mimics, critical underlying diseases and contraindications against standard systemic thrombolysis represent difficulties in IHS treatment. Quality of care may be improved by educating medical staff, implementing a code stroke and a CT-rendezvous system, providing access to specialized care (e.g. on stroke units) and endovascular reperfusion therapy as well as neuromonitoring, where applicable.
\end{abstract}

\section{Epidemiology and Definition}

Stroke is generally known to be among the leading causes for death and acquired disability worldwide ${ }^{1,2}$. It is less known that between $2.2 \%$ and $17 \%$ of all strokes occur in hospitalized patients, with a lower percentage reported in smaller hospitals and multicenter studies,4 and higher rate in tertiary clinics ${ }^{5,6}$. This difference is presumably due to a higher percentage of critically ill patients in larger hospitals, more frequent high-risk procedures and, possibly, a lower rate of reported cases in some participating centers in multicenter studies. In-hospital stroke (IHS) is most commonly defined as stroke, that occurs during hospitalization in a patient originally admitted for another diagnosis, provided that neuronal tissue damage due to infarction or hemorrhage is confirmed by cerebral imaging (usually computed tomography (CT) or magnetic resonance imaging (MRI) $)^{3,7}$. This definition usually excludes recurrent stroke ${ }^{8}$, progressive community-onset stroke (COS), global cerebral hypoperfusion and periprocedural complications of reperfusion therapies for patients with acute ischemic stroke (AIS). It includes complications of non-stroke related interventions such as percutaneous coronary interventions (PCI). Inpatient hemorrhagic stroke is widely regarded as subtype of IHS and has been reported to be less common than ischemic IHS (between $2 \%$ and $11 \%$ of all IHS) $)^{9,10}$.

\section{Etiology, Risk factors and Outcome}

Patients who are hospitalized for cardiac disease are especially vulnerable to IHS $^{7}$. Most ischemic IHS occur on cardiological or cardiosurgical wards ${ }^{11}$ after coronary artery bypass graft (CABG) or percutaneous coronary intervention (PCI) $)^{9,12}$ or, more generally, after invasive procedures $(70 \%)^{13}$. Accordingly, most patients with IHS are hospitalized because of myocardial infarction (MI) or $\mathrm{CABG}^{14}$. 
Stroke etiology according to TOAST criteria $^{15}$ is more often reported to be cardioembolic or undetermined in comparison to $\operatorname{COS}^{12,14}$. Inflammation, diabetes, dehydration, hypertension or instable blood-pressure, reduced renal function, reduced left ventricular ejection faction, newly detected atrial fibrillation and previous MI or stroke have been described as independent risk factors for IHS ${ }^{16-20}$. Although IHS patients generally have a higher vascular burden $^{3}$, the dominant etiology of IHS is cardioembolism. This special vulnerability may be the consequence of a general prothrombotic state due to inflammatory reactions and reduced organ function in critically ill patients or of necessary withdrawal of anticoagulation. The high incidence of IHS on cardiological wards suggests that direct injury or interventional manipulation of the heart further enhances the risk of cardioembolism. The closer in time the index event, the higher the IHS risk: four out of five strokes after MI occur during the first five days ${ }^{21}$. The absolute periprocedural or -operative risk to suffer from IHS is about $0.25 \%$ in $\mathrm{PCI}^{17}, 0.9 \%$ after acute coronary events $^{22}$ and about $1.4 \%$ after $\mathrm{CABG}^{23}$.

Truly iatrogenic IHS due to plaque rupture during PCI or accidental embolisation during cerebral angiography or coiling of aneurysms - approximately $11.8 \%$ of all IHS ${ }^{11}$ - are often difficult to determine, since the interventions themselves enhance the risk for cardioembolism. Most studies refer to the TOAST criteria for stroke etiology, which do not specifically identify iatrogenic causes. Thus, iatrogenic IHS may be underreported.

IHS is associated with unfavorable outcome. The mortality in IHS is higher than in COS, reaching up to $33 \%{ }^{11}$. Stroke after MI or CABG is a serious complication and increases in-hospital mortality significantly ${ }^{24,25}$. IHS patients are less likely to be discharged home $(27.7 \%$ (IHS) vs $49.9 \%$ (COS)) or to be functionally independent $(31.0 \%$ (IHS) vs $50.4 \%(\mathrm{COS}))^{3}$ and remain hospitalized for a longer time (mean 19.5 (IHS) vs 12.1 days (COS)) ${ }^{12}$. They are more likely to experience incontinence, dysphagia, a lower level of consciousness and higher grade of motor deficit ${ }^{26}$. The differences in the course of disease and outcome are foremost attributed to the underlying disease $\mathrm{e}^{5}$, but also to the greater stroke severity ${ }^{3}$ and possibly to a higher rate of large vessel occlusions (LVO) ${ }^{12}$ ) as well as due to delays in treatment.

\section{Diagnosis and Treatment}

Despite omission of the pre-hospital phase in IHS, longer time intervals between symptom recognition and start of reperfusion therapy ${ }^{9}$, as well as longer in-hospital delays $^{3,12}$ have been reported. While in COS patients symptoms are usually recognized by spouses or relatives, IHS is most often recognized by medical personnel $(80 \%)$, especially nurses $(64 \%)^{14}$. In case of an emergency call,
COS are usually taken to the emergency department by emergency medical services, where they are neurologically evaluated and immediately treated ${ }^{27}$. The COS rescue chain is a well-established multidisciplinary process, which constantly aims to reduce time intervals to ensure early diagnostics and treatment (e.g. pre-hospital notification of the neurologist before admission ${ }^{28}$ ). In IHS, however, stroke symptoms are often not recognized as an emergency ${ }^{11}$ and thus treated with significant delay. Since success of reperfusion treatment is time-dependent ${ }^{29}$ and must be initiated within the first hours after onset of symptoms, any delay can be detrimental to the patient's outcome ${ }^{7}$.

Besides reliable recognition of stroke symptoms, identification of onset time can be challenging, even in alert and otherwise healthy patients. Critically ill hospitalized patients, however, often are not able to notice and/or to communicate their deficits due to medication and/or underlying illness. Hence, IHS presents with reduced or altered level of consciousness more often than $\operatorname{COS}^{9,26}$. This may impair the neurological evaluation by the attending staff immensely, or render it impossible, even for specialists. Thus, despite professional medical surroundings, uncertainties regarding the time of symptom onset of IHS are frequently reported $(20 \%$ to $33,5 \%$ of patients $)^{7,12}$. The percentage of IHS with unknown onset is comparable to the one of COS (21\% wake-up stroke, $14 \%$ unknown onset) ${ }^{30}$.

Correct and timely diagnosis is furthermore complicated by a high rate of in-hospital stroke mimics. Sudden changes in mental status (most commonly seizures, hypotension or delirium) account for about $50 \%$ of suspected IHS ${ }^{31,32}$. This diagnostic uncertainty surely is in many cases the reason, why IHS workup is not adequate for a potentially treatable emergency ${ }^{14}$.

If IHS is recognized in time, various contraindications that are frequently present in hospitals, limit the use of standard reperfusion therapy with intravenous recombinant tissue plasminogen activator (IVRTPA). The most common contraindications are recent major surgery, current and sufficient anticoagulation, recent bleeding or terminal illness ${ }^{5,11}$. In patients who can receive IVRTPA, however, IVRTPA has been shown to be equally safe in IHS and in $\mathrm{COS}^{3}$. In recent years, endovascular approaches significantly improved the outcome of COS patients with LVO and should be considered whenever possible ${ }^{33}$. This holds even more true for IHS, where modern stent-assisted neurothrombectomy is a safe, effective and practicable treatment option ${ }^{12,34}$.

In COS, specialized neurological treatment on stroke units (SU) is well-established and proven to improve outcome and survival ${ }^{35}$. IHS are transferred to SU less often than $\operatorname{COS}^{10,26}$. This leads to a lower rate of adequate 
secondary prevention, assessment of deficits (e.g. dysphagia) and rehabilitation. Overall, IHS treatment shows lower adherence to treatment guidelines. For example, early use of rtPA, antithrombotic treatment, dysphagia screening and smoking cessation counselling were applied less often ${ }^{3}$.

\section{Recommended Treatment Approach}

Increased awareness, education and training of medical staff are fundamental for timely symptom recognition, especially on (identified) high risk wards, for example cardiologcial plus intensive and intermediate care units. While general knowledge about stroke symptoms is generally sufficient, specific knowledge about timedependent treatment options is often lacking ${ }^{36}$. Educational programs can enhance "code stroke" activations ${ }^{37}$. Patient with a high risk for IHS should be identified and closely clinically monitored for stroke symptoms (paralysis, numbness, diplopia, vision loss, chances in language and/or speech, non-orthostatic dizziness ${ }^{38}$ ). If possible, antithrombotic treatment should be started early $(<48 \mathrm{~h})$ after $\mathrm{MI}^{18}$ and paused anticoagulation resumed. After $\mathrm{CABG}$ the risk of early anticoagulation in case of atrial fibrillation, however, seems to outweigh the benefit ${ }^{39}$.

In case of suspected IHS, we recommend a clearly structured code stroke protocol. Protocol-driven approaches have been shown to significantly improve response times in $\operatorname{COS}^{40}$. In IHS, this has been achieved by appointing a specialized stroke response team that could be called by any staff member. By doing so, the median time from symptom recognition to CT could be reduced radically (74min compared to $271 \mathrm{~min}$ before the implementation $)^{41}$. The goal of a protocol-driven approach is to create unambiguous responsibilities and to perform tasks simultaneously instead of sequentially whenever possible (detailed pocket-card by E. Cumbler) ${ }^{42}$. If an acute IHS is suspected, we furthermore suggest a CTrendezvous system ${ }^{12}$ : the attending physician immediately contacts a vascular neurologist and neuroradiologist while transporting the patient to the CT and checking the relevant time points, medical history, contraindications against contrast agents and rtPA. The patient then receives a neurological evaluation and multimodal stroke CT at the (neuro-)radiological diagnostic site. In case of IHS, an interdisciplinary decision is then reached and treatment initiated. Due to contraindications against IVRTPA and a high rate of LVO in IHS, endovascular approaches should always be considered, especially in severely affected patients (NIHSS $\geq 7$ within 3 hours of symptom onset, NIHSS $\geq 9$ within 3-6 hours $)^{43}$. Hospitals without interventional stroke treatment options need to participate in neurovascular networks and establish pragmatic referral algorithms ${ }^{44}$.
Patients with IHS should, whenever possible, be treated on a SU or neurological ICU. Specialized stroke care is not limited to reperfusion therapy, but includes diagnostics and initiation of secondary prevention, physiotherapy, dysphagia assessment, logopedic treatment, rehabilitation assessment and planning as well as counselling for patients and family members ${ }^{45}$.

For patients undergoing procedures with high IHSrisk, neuromonitoring should be considered. Continuous EEG- and Doppler/Duplex ultrasound monitoring have been shown to effectively detect perioperative brain ischemia during vascular surgery ${ }^{46,47}$. However, these approaches have not routinely been established in longerterm monitoring due to personnel costs and practical constraints $^{48}$. Continuous EEG has been shown to reduce mortality on ICUs ${ }^{49}$, though, and could potentially overcome the limitations in clinical assessment of sedated and/or ventilated patients.

\section{Conclusion}

While generally regarded as the same disease, IHS differs from COS in distribution of etiology, risk factors and treatment options. Certain caveats demand special attention in recognizing and treating IHS. Staff education, a code stroke and CT-rendezvous system, specialized care and therapy and, in some cases, neuromonitoring are recommended to improve quality of care.

\section{References}

1. Lozano R, Naghavi M, Foreman K, et al. Global and regional mortality from 235 causes of death for 20 age groups in 1990 and 2010: A systematic analysis for the Global Burden of Disease Study 2010. The Lancet. 2012; 380(9859): 2095-2128. doi: 10.1016/S01406736(12)61728-0

2. Murray CJL, Vos T, Lozano R, et al. Disability-adjusted life years (DALYs) for 291 diseases and injuries in 21 regions 1990-2010 A systematic analysis for the Global Burden of Disease Study 2010. The Lancet. 2012; 380(9859): 2197-2223. doi: 10.1016/S01406736(12)61689-4

3. Cumbler E, Wald H, Bhatt DL et al. Quality of care and outcomes for in hospital ischemic stroke findings from the National Get With The Guidelines Stroke. Stroke. 2014; 45(1): 231-238. doi: 10.1161/ STROKEAHA.113.003617

4. Kimura K, Minematsu K, Yamaguchi T. Characteristics of in-hospital onset ischemic stroke. Eur Neurol. 2006; 55(3): 155-159. doi: 10.1159/000093574

5. Dulli D, Samaniego EA. Inpatient and community ischemic strokes in a university hospital. Neuroepidemiology. 2007; 28(2): 86-92. doi: 10.1159/000098551

6. Kelley RE, Kovacs AG. Mechanism of in-hospital cerebral ischemia. Stroke. 1986; 17(3): 430-433. doi: 10.1161/01.STR.17.3.430

7. Manawadu D, Choyi J, Kalra L. The impact of early specialist management on outcomes of patients with in-hospital stroke. PLoS ONE. 2014; 9(8): e104758. doi: 10.1371/journal.pone.0104758

8. Erdur H, Scheitz JF, Ebinger M, et al. In-hospital stroke recurrence and stroke after transient ischemic attack: frequency and risk factors. Stroke. 2015; 46(4): 1031-1037. doi: 10.1161/ STROKEAHA.114.006886 
9. Saltman AP, Silver FL, Fang J, et al. Care and Outcomes of Patients With In-Hospital Stroke. JAMA Neurol. 2015; 72(7): 749-755. doi: 10.1001/jamaneurol.2015.0284

10. Briggs $\mathrm{R}$, McDonagh $\mathrm{R}$, Mahon $\mathrm{O}$, et al. In-hospital stroke: characteristics and outcomes. Ir Med J. 2015; 108(1): 24-25.

11. Alvaro LC, Timiraos J, Sadaba F. In-hospital stroke: clinical profile and expectations for treatment Accidentes cerebrovasculares intrahospitalarios: perfil clinico y expectativas terapeuticas. Neurologia. 2008; 23(1): 4-9.

12. Schurmann K, Nikoubashman O, Falkenburger B, et al. Risk profile and treatment options of acute ischemic in-hospital stroke. J Neurol. 2016; 263(3): 550-557. doi: 10.1007/s00415-015-8010-2

13. Farooq MU, Reeves MJ, Gargano J, et al. In-hospital stroke in a statewide stroke registry. Cerebrovasc Dis. 2008; 25(1-2): 12-20. doi 10.1159/000111494

14. Alberts MJ, Brass LM, Perry A, et al. Evaluation times for patients with in-hospital strokes. Stroke. 1993; 24(12): 1817-1822.

15. Adams HP, Bendixen BH, Kappelle LJ, et al. Classification of subtype of acute ischemic stroke Definitions for use in a multicenter clinical trial TOAST Trial of Org 10172 in Acute Stroke Treatment. Stroke. 1993 24(1): 35-41. doi: 10.1161/01.STR.24.1.35

16. Nadav L, Gur AY, Korczyn AD, et al. Stroke in hospitalized patients are there special risk factors. Cerebrovasc Dis. 2002; 13(2): 127-131.

17. Dukkipati S, O'Neill WW, Harjai KJ, et al. Characteristics of cerebrovascular accidents after percutaneous coronary interventions. J Am Coll Cardiol. 2004; 43(7): 1161-1167. doi: 10.1016/j jacc.2003.11.033

18. Hachet O, Guenancia C, Stamboul K, et al. Frequency and predictors of stroke after acute myocardial infarction: specific aspects of in hospital and postdischarge events. Stroke. 2014; 45(12): 3514-3520. doi: 10.1161/STROKEAHA.114.006707

19. Guenancia C, Hachet 0 , Stamboul K, et al. Incremental predictive value of mean platelet volume platelet count ratio in in-hospital stroke after acute myocardial infarction. Platelets. 2016; 1-6. doi: 10.1080/09537104.2016.1203397

20. Pi Y, Zhang W, Shi L, et al. Study on the incidence and risk factors for in-hospital stroke in patients with acute myocardial infarction. Zhonghua Liu Xing Bing Xue Za Zhi. 2002; 23(6): 457-460

21. Hachet $\mathrm{O}$, Guenancia C, Stamboul $\mathrm{K}$, et al. Frequency and predictors of stroke after acute myocardial infarction specific aspects of in-hospital and postdischarge events. Stroke. 2014; 45(12): 3514-3520. doi: 10.1161/STROKEAHA.114.006707

22. Budaj A, Flasinska K, Gore JM, et al. Magnitude of and risk factors for in-hospital and postdischarge stroke in patients with acute coronary syndromes: findings from a Global Registry of Acute Coronary Events. Circulation. 2005; 111(24): 3242-3247. doi: 10.1161/ CIRCULATIONAHA.104.512806

23. Hornero F, Martín E, Rodríguez R, et al. A multicentre Spanish study for multivariate prediction of perioperative in-hospital cerebrovascular accident after coronary bypass surgery the PACK2 score. Interact Cardiovasc Thorac Surg. 2013; 17(2): 353. doi: 10.1093/icvts/ivt102

24. McKhann GM, Grega MA, Borowicz LM, et al.Stroke and encephalopathy after cardiac surgery: an update. Stroke. 2006; 37(2): 562-571. doi: 10.1161/01.STR.0000199032.78782.6c

25. Albaker O, Zubaid M, Alsheikh Ali AA, et al. Early stroke following acute myocardial infarction: incidence, predictors and outcome in six Middle-Eastern countries. Cerebrovasc Dis. 2011; 32(5): 471-482. doi: 10.1159/000330344

26. Bhalla A, Smeeton N, Rudd AG, et al. A comparison of characteristics and resource use between in-hospital and admitted patients with stroke. J Stroke Cerebrovasc Dis. 2010; 19(5): 357-363.
27. Patel MD, Rose KM, O'Brien EC, et al. Prehospital notification by emergency medical services reduces delays in stroke evaluation: findings from the North Carolina stroke care collaborative. Stroke. 2011; 42 (8): 2263-2268. doi: 10.1161/STROKEAHA.110.605857

28. Casolla B, Bodenant M, Girot M, et al. Intra-hospital delays in stroke patients treated with rt-PA: impact of preadmission notification. J Neurol. 2013; 260(2): 635-639. doi: 10.1007/s00415-012-6693-1

29. Menon BK, Almekhlafi MA, Pereira VM, et al. Optimal workflow and process-based performance measures for endovascular therapy in acute ischemic stroke: analysis of the Solitaire FR thrombectomy for acute revascularization study. Stroke. 2014; 45(7): 2024-2029. doi: 10.1161/STROKEAHA.114.005050

30. Reid JM, Dai D, Cheripelli B, et al. Differences in wake-up and unknown onset stroke examined in a stroke registry. Int J Stroke. 2015; 10(3): 331-335. doi: 10.1111/ijs.12388

31. El Husseini N, Goldstein LB. "Code stroke": hospitalized versus emergency department patients. J Stroke Cerebrovasc Dis. 2013; 22(4): 345-348. doi: 10.1016/j.jstrokecerebrovasdis.2011.09.012

32. Cumbler E, Simpson J. Code stroke Multicenter experience with inhospital stroke alerts. J Hosp Med. 2014. doi: 10.1002/jhm.2311

33. Powers WJ, Derdeyn CP, Biller J, et al. 2015 American Heart Association/American Stroke Association Focused Update of the 2013 Guidelines for the Early Management of Patients With Acute Ischemic Stroke Regarding Endovascular Treatment: A Guideline for Healthcare Professionals From the American Heart Association/ American Stroke Association. Stroke. 2015; 46(10): 3020-3035. doi: 10.1161/STR.0000000000000074

34. Yoo J, Song D, Lee K, et al. Comparison of Outcomes after Reperfusion Therapy between In-Hospital and Out-of-Hospital Stroke Patients. Cerebrovasc Dis. 2015; 40(1-2): 28-34. doi: 10.1159/000381787

35. Stroke Unit Trialists' Collaboration Organised inpatient (stroke unit) care for stroke. Cochrane Database Syst Rev. 2007; (4): CD000197. doi: 10.1002/14651858.CD000197.pub2

36. Mellon L, Hasan H, Lee S, et al. Knowledge of Thrombolytic Therapy Amongst Hospital Staff: Preliminary Results and Treatment Implications. Stroke. 2015; 46(12): 3551-3553. doi: 10.1161/ STROKEAHA.115.010327

37. Rodríguez Campello A, Cuadrado Godia E, Giralt Steinhauer E, et al. Detección de ictus intrahospitalario: evaluación de resultados de un programa de formación y entrenamiento a personal médico y de enfermería (Detecting in-hospital stroke: Assessment of results from a training programme for medical personnel). Neurologia. 2014. doi: 10.1016/j.nrl.2014.06.003

38. Goldstein LB, Simel DL. Is this patient having a stroke. JAMA. 2005; 293(19): 2391-2402. doi: 10.1001/jama.293.19.2391

39. Schulman S, Cybulsky I, Delaney J. Anticoagulation for stroke prevention in new atrial fibrillation after coronary artery bypass graft surgery. Thromb Res 2015. doi: 10.1016/j.thromres.2015.02.015

40. Rai AT, Smith MS, Boo S, et al. The 'pit-crew' model for improving door-to-needle times in endovascular stroke therapy: a Six-Sigma project. J Neurointerv Surg. 2016; 8(5): 447-452. doi: 10.1136/ neurintsurg-2015-012219

41. Cumbler E, Anderson T, Neumann R, et al. Stroke alert program improves recognition and evaluation time of in-hospital ischemic stroke. J Stroke Cerebrovasc Dis. 2010; 19(6): 494-496. doi: 10.1016/j.jstrokecerebrovasdis.2009.09.007

42. Cumbler E. In-Hospital Ischemic Stroke. Neurohospitalist. 2015; 5(3): 173-181. doi: 10.1177/1941874415588319

43. Heldner MR, Zubler C, Mattle HP, et al. National Institutes of Health stroke scale score and vessel occlusion in 2152 patients with acute ischemic stroke. Stroke. 2013; 44(4): 1153-1157. doi: 10.1161/ STROKEAHA.111.000604 
44. Weber R, Reimann G, Weimar C, et al. Outcome and periprocedural time management in referred versus directly admitted stroke patients treated with thrombectomy. Ther Adv Neurol Disord. 2016; 9(2): 7984. doi: $10.1177 / 1756285615617081$

45. Lawrence M, Pringle J, Kerr S, et al. Multimodal secondary prevention behavioral interventions for TIA and stroke: a systematic review and meta-analysis. PLoS ONE. 2015; 10(3): e0120902. doi: 10.1371/ journal.pone.0120902

46. Ballotta E, Saladini M, Gruppo M, et al. Predictors of electroencephalographic changes needing shunting during carotid endarterectomy. Ann Vasc Surg. 2010; 24(8): 1045-1052. doi: 10.1016/j.avsg.2010.06.005
47. Wang X, Ji B, Yang B, et al. Real-time continuous neuromonitoring combines transcranial cerebral Doppler with near-infrared spectroscopy cerebral oxygen saturation during total aortic arch replacement procedure: a pilot study. ASAIO J. 2012; 58(2): 122-126. doi: 10.1097/MAT.0b013e318241abd3

48. Young GB, Campbell VC. EEG monitoring in the intensive care unit: pitfalls and caveats. J Clin Neurophysiol. 1999; 16(1): 40-45.

49. Ney JP, van der Goes DN, Nuwer MR, et al. Continuous and routine EEG in intensive care: utilization and outcomes, United States 2005-2009. Neurology. 2013; 81(23): 2002-2008. doi: 10.1212/01. wnl.0000436948.93399.2a 